\title{
ESTADO ACTUAL DE LAS POBLACIONES DE FUCUS VESICULOSUS L. (ALGAE PHAEOPHYTA) EN LA RIA DE VIGO
}

\author{
J. P. PAZO y X. M. ROMARIS *
}

\section{RESUMEN:}

Se han estudiado las poblaciones de Fucus vesiculosus L. en tres estaciones de la Ría de Vigo en dos ocasiones: verano de 1975 y verano de 1978. Se efectuaron análisis biométricos, de número de vesículas, proporción de vesículas axilares y otros parámetros. Los datos obtenidos permiten afirmar que la "reseptentrionalización" de las costas gallegas se mantiene en el momento presente.

\section{SUMMARY:}

The populations of Fucus vesiculosus L. at three stations of Ría de Vigo was studied in two times: summer of 1975 and summer of 1978 . We make biometric analysis, number of vesicles, percentage of axillary, vesicles and other parameters. The dates obtained permits assert that the reseptentrionalisation of galician coasts is maintained at present moment.

\section{INTRODUCCION}

La gran mayoria de las especies del género Fucus están limitadas en su distribución al hemisferio norte; se trata de un género de distribución boreal, más particularmente boreal-atlántica (Levring, Hoppe, y Schmid, 1969).

Sobre las costas atlánticas europeas su distribución se extiende desde Escandinavia hasta las costas de Portugal, aunque $F$. vesiculosus alcanza las costas de $\mathrm{Ma}$ rruecos, en éstas se refugia en lagunas costeras y estuarios(Safi) (Gayral, 1958 y Fischer-Piette, 1961). En las Islas Canarias se ha encontrado una población de $F$. vesiculosus en Arrecife de Lanzarote (Davy de Virville y Denizot, 1958, y Fischer-Piette, 1961). 
Fucus vesiculosus es una especie que presenta gran variedad morfológica de acuerdo con las diferentes características de los distintos hábitats (Seoane-Camba, 1966). Así, en las proximidades del límite Norte de su distribución existe una variedad de $F$. vesiculosus de menor tamaño que la especie típica: es la var. palmatus; asimismo en las proximidades de la frontera Sur de la especie aparece una variedad descrita por Fischer-Piette: es la forma $F$. vesiculosus evesiculosus pendens fructuplanus atendiendo a: la carencia de vesículas (evesiculosus), a que penden de las rocas y tienen su tercio inferior reducido al nervio central (pendens), y a presentar los receptáculos planos como los de F. serratus (fructuplanus),

En la Ría de Vigo, la casi totalidad de los ejemplares de F. vesiculosus presentan todas o alguna de las características de la forma evesiculosus pendens fructuplanus. (J. P. Pazo, 1975).

Se ha especulado con la presencia de un presunto híbrido $F$. vesiculosus $x F$. serratus (Burrows y Lodge, 1952), (Seoane-Camba, 1966); según unos autores (Burrows y Lodge, 1952) este híbrido serviría para dar altura en la zona litoral a los ejemplares de $F$. serratus debido a la adquisición de genes de $F$. vesiculosus. Gracias a esto, $F$. serratus aumentaría la extensión de su cintura en sentido ascendente.

Para otros autores (Seoane-Camba, 1966) la existencia de este híbrido en ciertas áreas (las Rías Gallegas, por ejemplo) se debería a un efecto de frontera definido por Kniep, (Kniep, 1925). Según esto, en zonas de frontera, como son las Rías Bajas para $F$. serratus, la concurrencia del híbrido se vería favorecida frente a las especies parentales puras.

Nosotros consideramos improbable la existencia de este híbrido, y a falta de estudios genéticos serios, asimilamos los individuos que se le atribuyen a la forma evesiculosus pendens fructuplanus de Fischer-Piette.

\section{ANTECEDENTES}

Desde 1955 hasta nuestros días las poblaciones de F. vesiculosus de las Rías Gallegas vienen siendo estudiadas por muchos autores: en los años cincuenta son Fischer-Piette y F. Ardré, en los años sesenta es Seoane-Camba, y en los años setenta J. P. Pazó; ésto nos da una imagen de la evolución de las poblaciones en los últimos veinte años, de lo cual se pueden extraer interesantes conclusiones biogeográficas.

Hemos estudiado tres localidades bien definidas dentro de la Ría de Vigo, tomándose muestras en cada una de ellas en dos ocasiones: la primera durante el verano de 1975 y la segunda en el mes de Septiembre de 1978, tratando de seguir la evolución de las poblaciones mediante un análisis similar al efectuado por SeoaneCamba (1966).

Desde los primeros trabajos de Fischer-Piette hasta la actualidad se ha venido observando un progresivo acercamiento de las poblaciones de F. vesiculosus a las 
condiciones óptimas de la especie, que se advierte por un aumento de la talla media de las poblaciones y por la presencia de vesículas y hemivesículas indicativas de una creciente revesiculización; esta mejora de las condiciones de $F$. vesiculosus en las Rías Gallegas, la interpretamos siguiendo a Fischer-Piette como un indicio de una «reseptentrionalización» del bios de las costas gallegas que viene teniendo lugar desde los años cincuenta hasta el presente.

\section{METODOS}

En este trabajo hemos estudiado las poblaciones de $F$. vesiculosus, en tres puntos de la Ría, cada uno de ellos con unas condiciones características: una primera estación, situada en Arcade, en la parte más interna de la Ría, zona plenamente estuárica, con aguas de baja salinidad, en una zona muy protegida y donde $F$. vesiculosus crece sobre rocas parcialmente cubiertas de fango en las que aparecen una serie de variedades limícolas de la especie que estudiamos más ampliamente en otro trabajo (X. Niell y J.P. Pazo, en prensa).

Otra de las estaciones visitadas es Coruxo, en la orilla sur de la Ría y en su parte central, un enclave ya claramente oceánico, zona semiexpuesta y donde $F$. vesiculosus está representado casi exclusivamente por ejemplares de la forma evesiculosus pendens fructuplanus.

La tercera estación visitada es una estación claramente oceánica, situada en las Islas Cies, a la entrada de la Ría; es un enclave poco expuesto y donde $F$. vesiculosus presenta la población más floreciente de la Ría, con ejemplares excepcionalmente vesiculados y con una talla similar a la que presenta la especie en las costas inglesas del Canal de la Mancha. (Knight y Parke, 1950), (fig. 2).

\section{RESULTADOS}

I.-En Arcade la población de F. vesiculosus presenta ejemplares típicos de zonas de aguas salobres, con ejemplares de talo espiralizado, con existencia de largas vesículas laterales ("boursuflores»), con fronde de consistencia papirácea y fructificaciones muy estrechas y divaricadas.

En el verano de 1975, la talla media era de 16,05 cm., con una desviación típica de 6,39; en el verano de 1978 la talla media era $15,81 \mathrm{~cm}$., y su desviación típica 7,09 .

2.-En Coruxo, zona semiexpuesta y donde la totalidad de los individuos pertenecen a la variedad evesiculosus pendens fructuplanus, en el verano de 1975 la talla media era de 17,60 cm., y una desviación típica de 6,61; en el verano de 1978, la talla media fue de 18,74 cm., con una desviación típica de 6,53.

3.-En la Ensenada del Lago, Islas Cies, es donde las poblaciones de F. vesiculosus presentan un aspecto mas floreciente, alcanzando tallas propias de una latitud 


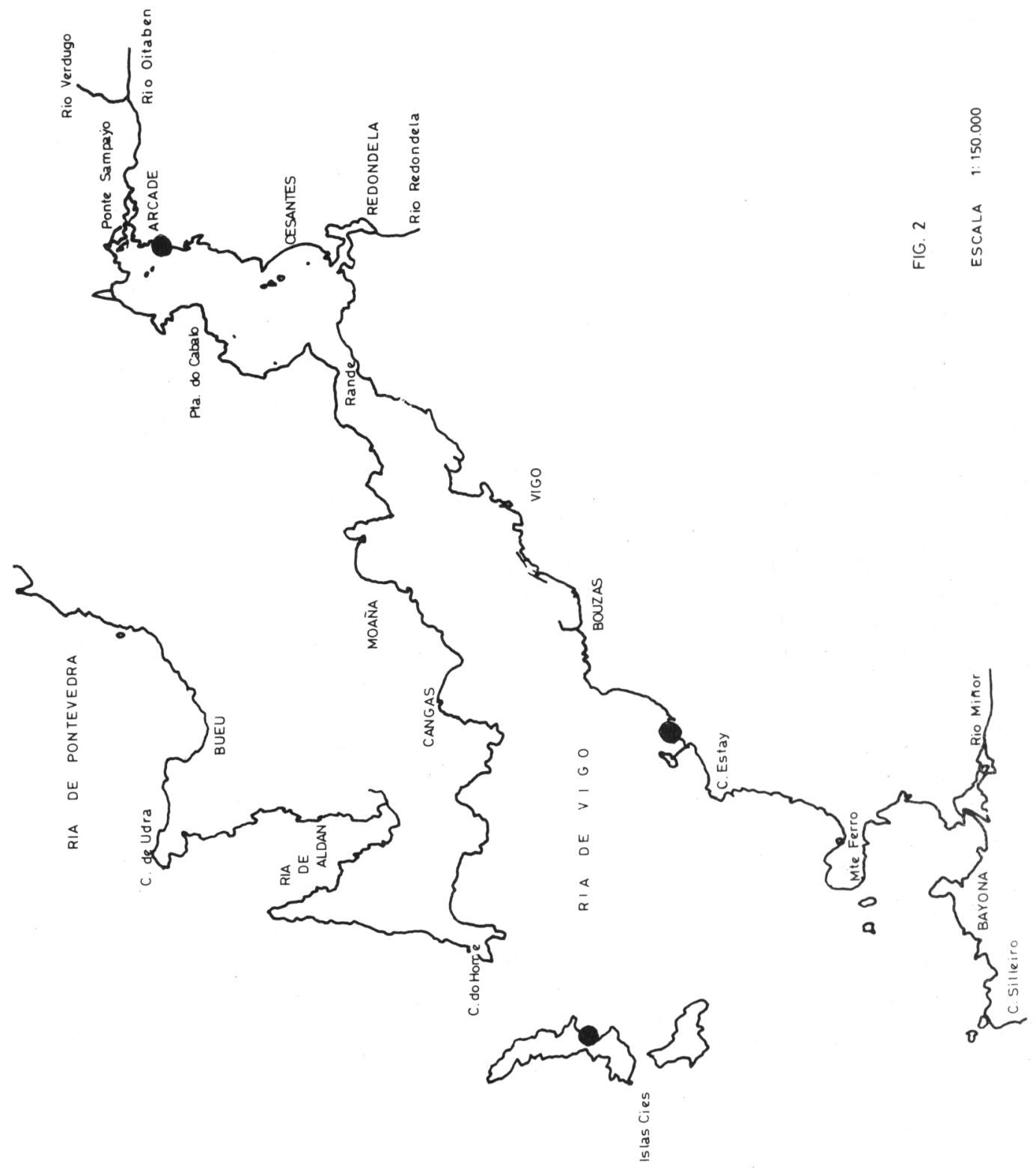


mas nórdica, donde la especie presenta sus poblaciones mejor constituidas. En el verano de 1975 la talla media era de $38,26 \mathrm{~cm}$., con una desviación típica de 14,94; en el verano de 1978 la talla media fue de 32,54 cm., y su desviación típica 14,02.

4.-En el verano de 1975 la población de Arcade presentaba un 70,42\% de individuos vesiculados. En 1978 la proporción era de 44,30\%.

5.-La población de Coruxo mostraba una proporción de individuos vesiculados del $18 \%$ en 1975. En el verano de 1978 esta proporción era del 22,62\%.

6.-En 1975 en las Islas Cies se observó una vesiculización que alcanzaba el 95,93 \% de los ejemplares. En 1978 esta proporción fue de un 92,76\%.

7.-El análisis comparativo de las tallas de las poblaciones mediante el estadístico Z en los años 1975 y 1978 nos da:

Para las poblaciones de Arcade, $\mathrm{Z}=0,26 \ll 1,96$

Para las poblaciones de Coruxo, $Z=+1,54 \ll 1,96$

Para las poblaciones de las Islas Cies, $\mathrm{Z}=3,24 » 1,96$

\section{CONCLUSIONES}

I.-Las poblaciones de Arcade en los años 1975 y 1978, y las de Coruxo, no son significativamente distintas con un margen de confianza del 95\%, mientras que las poblaciones de las Islas Cies en los años 1975 y 1978 son significativamente distintas al $95 \%$ de confianza. (Fig. 3).

2.-La revesiculización disminuye en Arcade entre 1975 y 1978 y se mantiene en Coruxo e Islas Cies. La menor proporción de individuos vesiculados en Arcade 78 puede deberse al mayor tamaño de muestra y a un muestreo mas exhaustivo que el realizado en 1975 .

3.-A la vista de los análisis biométricos realizados en 1975 y 1978 así como de los porcentajes de individuos vesiculados, presencia de vesiculas axilares y hemivesiculas, podemos concluir que la revesiculización de la especie en las Rías asi como la reseptentrionalización observada por Fischer-Piette desde los años 50 se mantiene y no se observa un incremento importante en los últimos tres años, con lo que podemos suponer que las poblaciones de $F$. vesiculosus están estabilizadas, o alcanzando la estabilidad en la Ría de Vigo. 

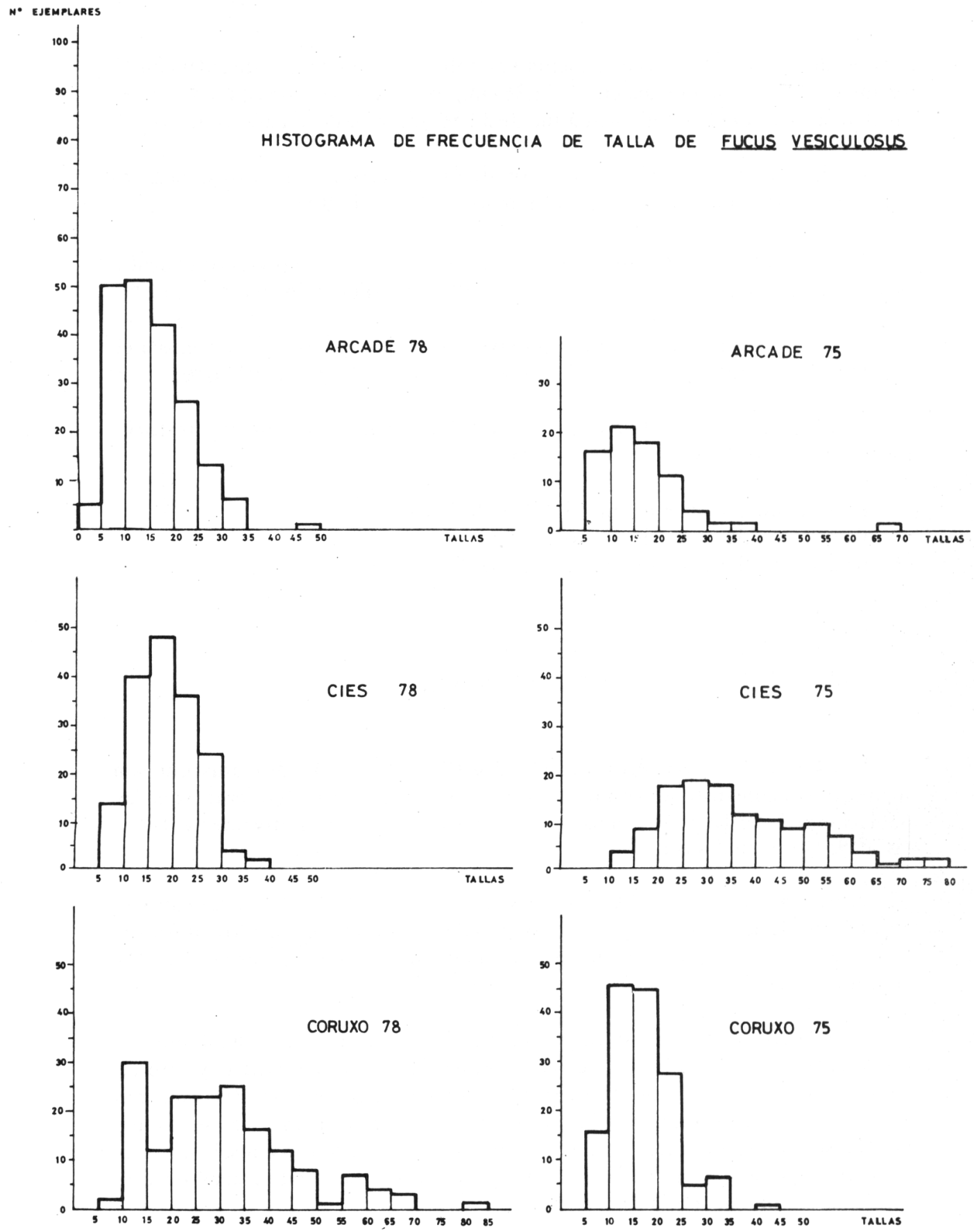

FIG. 3 
Datos del análisis de $F$. vesiculosus en Arcade.

\begin{tabular}{|c|c|c|c|c|c|c|c|c|c|}
\hline 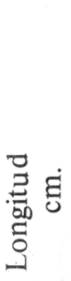 & 总: & 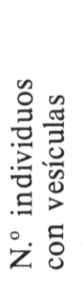 & 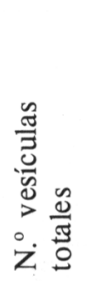 & 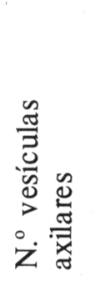 & 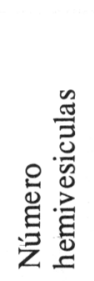 & 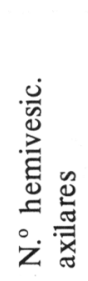 & 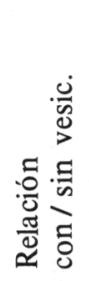 & 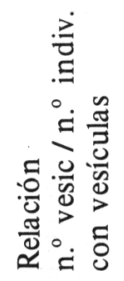 & 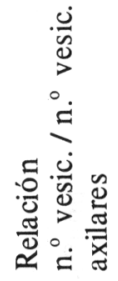 \\
\hline 5 & 5 & & & & & & 0 & & \\
\hline 6 & 4 & & & & & & 0 & & \\
\hline 7 & 7 & & & & & & 0 & & \\
\hline 8 & 4 & & & & & & 0 & & \\
\hline 9 & 13 & 2 & 8 & 2 & & & 0,18 & 4 & 4 \\
\hline 10 & 22 & 8 & 37 & 32 & & & 0,57 & 4,63 & 1,16 \\
\hline 11 & 12 & 3 & 74 & 74 & & & 0,33 & 24,67 & 1 \\
\hline 12 & 16 & 6 & 67 & 27 & & & 0,60 & 11,17 & 2,48 \\
\hline 13 & 9 & 4 & 14 & 14 & & & 0,8 & 3,5 & 1 \\
\hline 14 & 7 & 2 & 11 & 8 & & & 0,4 & 5,5 & 1,38 \\
\hline 15 & 7 & 4 & 16 & 11 & & & 1,33 & 4 & 1,45 \\
\hline 16 & 7 & 5 & 44 & 37 & 1 & & 2,5 & 8,8 & 1,19 \\
\hline 17 & 5 & 5 & 85 & 54 & & & - & 17 & 1,57 \\
\hline 18 & 13 & 7 & 46 & 25 & & & 1,17 & 6,57 & 1,84 \\
\hline 19 & 6 & 4 & 85 & 58 & & & 2 & 21,25 & 1,47 \\
\hline 20 & 11 & 3 & 35 & 33 & & & 0,38 & 11,67 & 1,06 \\
\hline 21 & 5 & 1 & 1 & 1 & & & 0,25 & 1 & 1 \\
\hline 22 & 7 & 3 & 31 & 12 & & & 0,75 & 10,33 & 2,58 \\
\hline 23 & 5 & 3 & 11 & 7 & & & 1,5 & 3,67 & 1,57 \\
\hline 24 & 4 & 4 & 161 & 96 & & & - & 40,25 & 1,68 \\
\hline 25 & 5 & 3 & 40 & 13 & & & 1,5 & 13,33 & 3,08 \\
\hline 26 & 5 & 3 & 53 & 5 & & & 1,5 & 17,67 & 10,6 \\
\hline 27 & 3 & 2 & 6 & 6 & & & 2 & 3 & 1 \\
\hline 28 & 3 & 1 & 9 & 6 & & & 0,5 & 9 & 1,5 \\
\hline 29 & 1 & 1 & 1 & 1 & & & - & 1 & 1 \\
\hline 30 & 1 & 1 & 9 & 8 & & & - & 9 & 1,13 \\
\hline 31 & 2 & 2 & 19 & 17 & & & - & 9,5 & 1,12 \\
\hline \multicolumn{10}{|l|}{32} \\
\hline 33 & 3 & 2 & 6 & 4 & & & 2 & 3 & 1,5 \\
\hline 34 & 1 & 1 & 45 & 34 & & & - & 45 & 1,32 \\
\hline \multicolumn{10}{|l|}{35} \\
\hline \multirow{2}{*}{\multicolumn{10}{|c|}{ - }} \\
\hline & & & & & & & & & \\
\hline 47 & 1 & 1 & 7 & 4 & & & - & 7 & 1,75 \\
\hline
\end{tabular}


Datos del análisis de $F$. vesiculosus en Coruxo.

\begin{tabular}{|c|c|c|c|c|c|c|c|c|c|}
\hline 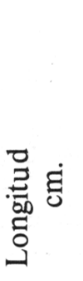 & 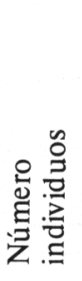 & 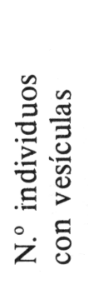 & 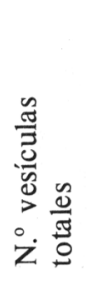 & 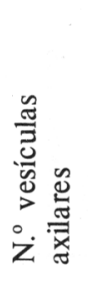 & 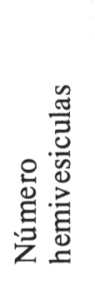 & 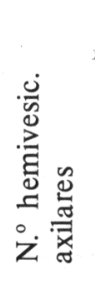 & 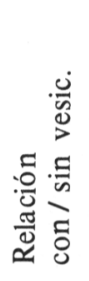 & 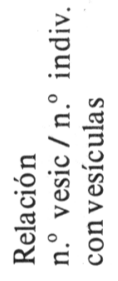 & 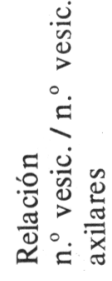 \\
\hline 6 & 3 & & & & & & 0 & & \\
\hline 7 & 3 & & & & & & 0 & & \\
\hline 8 & 3 & 1 & 2 & 2 & & & 0,50 & 2 & 1 \\
\hline 9 & 3 & & & & & & 0 & & \\
\hline 10 & 2 & & & & & & 0 & & \\
\hline 11 & 6 & 1 & 1 & 1 & 1 & 1 & 0,20 & 1 & 1 \\
\hline 12 & 8 & 3 & 14 & 12 & 1 & 1 & 0,60 & 4,67 & 1,17 \\
\hline 13 & 10 & 2 & 3 & 3 & 1 & 1 & 0,25 & 1,50 & 1 \\
\hline 14 & 8 & 2 & 3 & 3 & & & 0,33 & 1,50 & 1 \\
\hline 15 & 8 & 2 & 13 & 13 & 2 & 2 & 0,33 & 6,50 & 1 \\
\hline 16 & 15 & 3 & 11 & 11 & & & 0,25 & 3,67 & 1 \\
\hline 17 & 11 & 2 & 6 & 6 & 4 & 4 & 0,22 & 3 & 1 \\
\hline 18 & 9 & 3 & 66 & 37 & 8 & 8 & 0,50 & 22 & 1,78 \\
\hline 19 & 7 & 2 & 2 & 2 & & & 0,40 & 1 & 1 \\
\hline 20 & 6 & 2 & 24 & 22 & 7 & 4 & 0,50 & 12 & 1,09 \\
\hline 21 & 11 & 4 & 17 & 17 & 7. & 7 & 0,57 & 4,25 & 1 \\
\hline 22 & 12 & 4 & 21 & 21 & 5 & 5 & 0,50 & 5,25 & 1 \\
\hline 23 & 9 & & & & 11 & 11 & 0 & & \\
\hline 24 & 1 & & & & 2 & 2 & 0 & & \\
\hline 25 & 3 & & & & 1 & 1 & 0 & & \\
\hline 26 & 5 & 3 & 17 & 15 & 2 & 2 & 1,50 & 5,67 & 1,13 \\
\hline 27 & 5 & 1 & 1 & 1 & 2 & 2 & 0,25 & 1 & 1 \\
\hline 28 & 7 & 1 & 7 & 7 & 20 & 18 & 0,17 & 7 & 1 \\
\hline 29 & 6 & & & & & & 0 & & \\
\hline 30 & 1 & 1 & 1 & 1 & & & - & 1 & 1 \\
\hline 31 & 2 & & & & & & 0 & & \\
\hline 32 & & & & & & & & & \\
\hline 33 & 1 & 1 & 7 & 7 & 4 & 4 & - & 7 & 1 \\
\hline 34 & & & & & & & & & \\
\hline 35 & 1 & & & & & & 0 & & \\
\hline 36 & & & & & & & & & \\
\hline 37 & 1 & & & & & & 0 & & \\
\hline 38 & & & & & & & & & \\
\hline 39 & 1 & & & & & & 0 & & \\
\hline 40 & & & & & & & & & \\
\hline
\end{tabular}


Datos del análisis de $F$. vesiculosus de las Islas Cies.

\begin{tabular}{|c|c|c|c|c|c|c|c|c|c|}
\hline 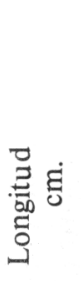 & 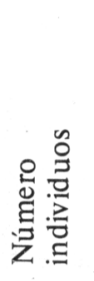 & 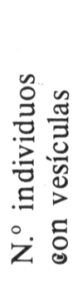 & 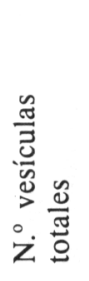 & 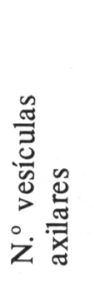 & 章 & 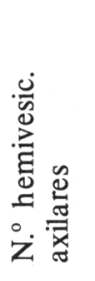 & 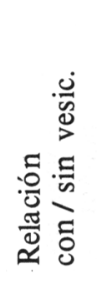 & 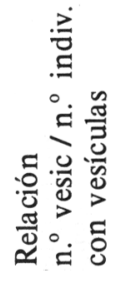 & 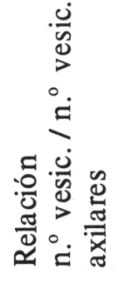 \\
\hline 9 & 1 & & & & & & 0 & & \\
\hline 10 & 1 & & & & & & 0 & & \\
\hline 11 & & & & & & & & & \\
\hline 12 & & & & & & & & & \\
\hline 13 & 5 & 2 & 7 & 6 & & & 0,67 & 3,5 & 1,2 \\
\hline 14 & 4 & 3 & 9 & 7 & 2 & 2 & 3 & 3 & 1,3 \\
\hline 15 & 6 & 6 & 78 & 42 & 2 & 2 & - & 13 & 1,9 \\
\hline 16 & 2 & 1 & 7 & 7 & 5 & 5 & 1 & 7 & 1 \\
\hline 17 & 1 & 1 & 21 & 12 & & & - & 21 & 1,8 \\
\hline 18 & 3 & 3 & 15 & 10 & 7 & 3 & - & 5 & 1,5 \\
\hline 19 & 3 & 2 & 26 & 10 & 2 & 1 & 2 & 13 & 2,6 \\
\hline 20 & 3 & 3 & 30 & 21 & 3 & 3 & - & 10 & 1,4 \\
\hline 21 & 3 & 3 & 36 & 34 & & & - & 12 & 1,1 \\
\hline 22 & 5 & 5 & 73 & 56 & 9 & 6 & - & 14,6 & 1,3 \\
\hline 23 & 5 & 5 & 160 & 57 & 12 & 5 & - & 32 & 2,81 \\
\hline 24 & 7 & 6 & 139 & 100 & 15 & 12 & 6 & 23,2 & 1,39 \\
\hline 25 & 3 & 3 & 111 & 59 & 6 & 1 & - & 37 & 1,88 \\
\hline 26 & 5 & 5 & 113 & 91 & 13 & 7 & - & 22,6 & 1,24 \\
\hline 27 & 5 & 4 & 124 & 63 & 27 & 21 & 4 & 31 & 1,97 \\
\hline 28 & 5 & 5 & 81 & 59 & & & - & 16,2 & 1,37 \\
\hline 29 & 4 & 4 & 134 & 97 & 4 & 4 & - & 33,5 & 1,38 \\
\hline 30 & 4 & 4 & 229 & 133 & 14 & 8 & - & 57,2 & 1,72 \\
\hline 31 & 6 & 6 & 455 & 230 & 74 & 31 & - & 75,8 & 1,98 \\
\hline 32 & 4 & 4 & 128 & 80 & & & - & 32 & 1,60 \\
\hline 33 & 5 & 5 & 329 & 214 & 4 & 4 & - & 65,8 & 1,54 \\
\hline 3 & 6 & 6 & 236 & 164 & & & - & 39,3 & 1,44 \\
\hline 35 & 4 & 4 & 111 & 54 & 3 & 1 & - & 27,8 & 2,06 \\
\hline 36 & 3 & 3 & 233 & 136 & 33 & 12 & - & 77,7 & 1,71 \\
\hline 37 & 3 & 3 & 203 & 116 & 7 & 3 & - & 67,7 & 1,75 \\
\hline 38 & 3 & 3 & 70 & 45 & 5 & 5 & - & 23,3 & 1,56 \\
\hline 39 & 5 & 5 & 168 & 136 & & & - & 33,6 & 1,24 \\
\hline 40 & 2 & 2 & 97 & 45 & 3 & 1 & - & 48,5 & 2,16 \\
\hline 41 & 4 & 4 & 204 & 164 & & & - & 51 & 1,24 \\
\hline 42 & 1 & 1 & 108 & 70 & & & - & 108 & 1,54 \\
\hline 43 & 3 & 3 & 382 & 283 & 2 & & - & 127,3 & 1,35 \\
\hline 44 & 1 & 1 & 49 & 38 & & & - & 49 & 1,29 \\
\hline 45 & 3 & 3 & 212 & 112 & 2 & 2 & - & 70,7 & 1,89 \\
\hline 46 & 2 & 2 & 59 & 53 & & & - & 29,5 & 1,11 \\
\hline
\end{tabular}


Datos del análisis de $F$. vesiculosus de las Islas Cies. (Continuación).

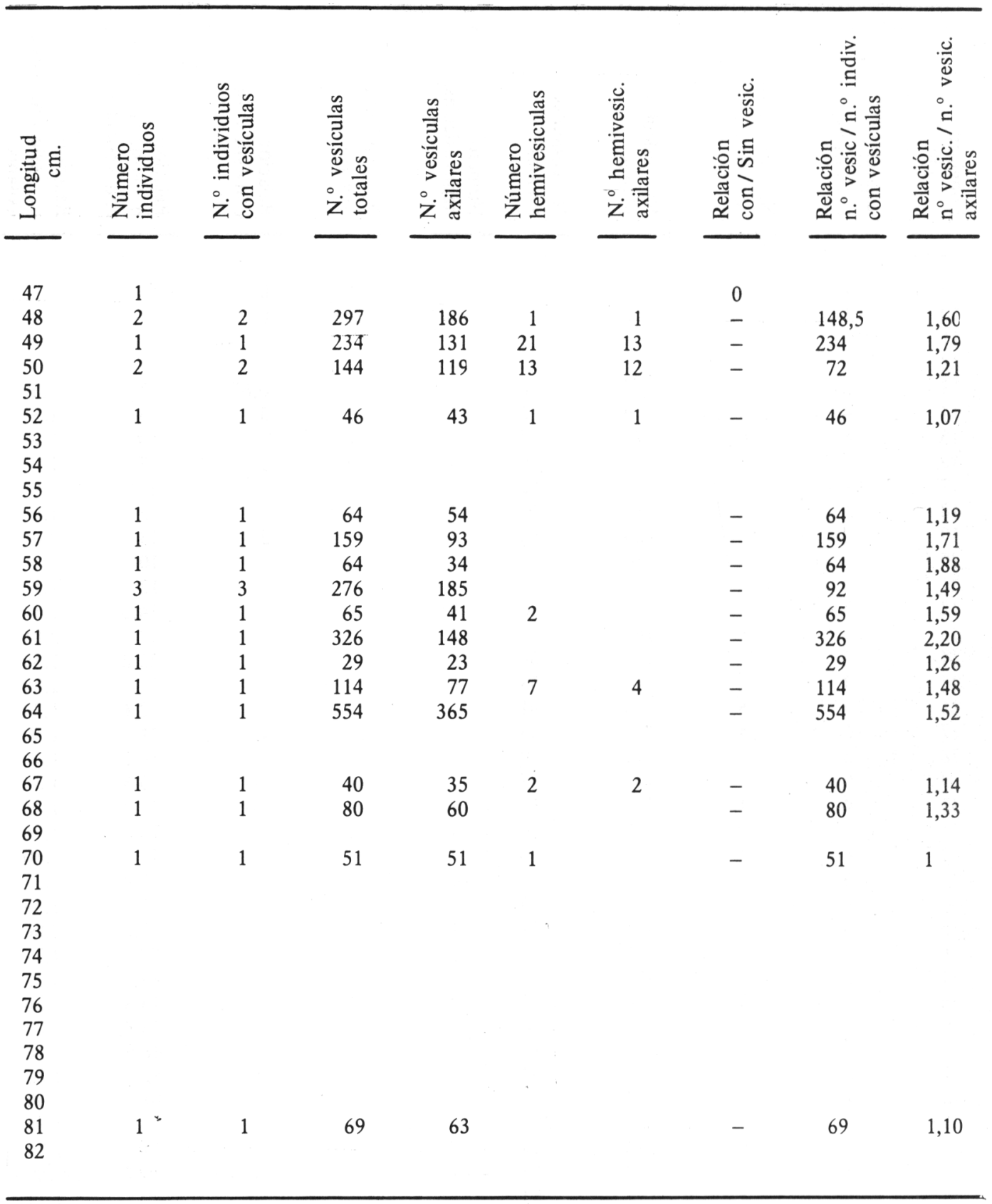




\section{AGRADECIMIENTOS}

Agradecemos al Sr. Gerente del Plan Marisquero de Galicia, D. Manuel Gonzalez Vidal las facilidades prestadas para la realización de este trabajo. A nuestros compañeros Francisco Fernández Cortes y Rosa Ramonell les agradecemos su colaboración en diversas partes de trabajo.

\section{BIBLIOGRAFIA}

ARDRE, F. 1957. Florule hivernale de la Ría de Vigo, Rev. Algologique, 3, 135-146.

ARDRE, F. 1970 Contribution a l'étude des algues marines de Portugal, I. La Flore. Portugal Acta Biológica 10 (1-4).

ARDRE, F. 1971. Contributión a l'étude des algues marines de Portugal, II. Ecologie et chorologie, Bull. Cent. Etud. Rech. Schi., 8 (3): 359-574. Biarritz.

ARDRE, F., F. CABAÑAS-RUESCAS, E. FISCHER-PIETTE y J. SEONE. 1958. Petite contribution a une monographie bionomique de la Ría de Vigo, Bullet. de l'Institut Oceanographique, 30, Mónaco.

BURROWS, E. and LODGE, Sh. 1952. Species problems in Fucus, Jour, Mar. Biol. Ass., 30: 161-176.

FISCHER-PIETTE, E. 1961. Sur l'écologie de la non vesiculisation du Fucus vesiculosus L., Rev. Gen. de Botanique, 68: 302-315.

FISCHER-PIETTE., et. F. ARDRE. 1967. Nouvelles remarques sur la revesiculisation du Fucus vesiculosus var evesiculosus, Rev. Algologique, 4: 289-301.

FISCHER-PIETTE et R. DUPERIER. 1960. Variations des fucacees de la Côte Basque de 1894 a 1959, Bull. Cent. Etud. Rech. Sci., 3 (1): 67-90. Biarritz.

GAYRAL, P. 1958. Algues de la Côte Atlantique Marocaine. Rabat.

GONTRAN HAMEL. 1939. Pheophycées de France. París.

JORDAN, A. J. and VADAS, R. L. 1972. Influence of environmental parameters on intraspecific variation in Fucus vesiculosus., Marine Biology, 14, (3): 248-252.

KNIGHT, M. and PARKE, M. 1950. A biological study of Fucus vesiculosus and Fucus serratus. Journal of Marine Biological Association, 29: 493-514.

LEVRING, HOPPE and SCHMID. 1969. Marine Algae. Von Springer Verlag. Hamburgo.

LEWIS, J. R. 1964. The ecology of rocky shores, The English Univ. Press. Ltd. London.

PAZO, J. P. 1975. Autoecología y distribución de las especies del género Fucus L. en la Ría de Vigo, Tesina Universidad de Santiago.

PAZO, J. P., y NIELL, X. 1977. Distribución y características de Fucus serratus L. en las Rías Bajas gallegas, Inv. Pesquera, 41 (2), Barcelona.

SAIZ, F., LOPEZ-BENITO, M., y ANADON, E. Estudio hidrográfico de la Ría de Vigo, Inv. Pesquera, VIII, 1957, 30-87; XVIII, 1961, 97-133; XX, 1961, 83-130. Barcelona.

SEOANE-CAMBA, J. 1965. Estudio sobre las algas bentónicas en la costa Sur de la Península Ibérica, Inv. Pesquera 23: 3-216. Barcelona.

SEOANE-CAMBA, J. 1966. Sobre la variabilidad morfológica de Fucus vesiculosus en las Rías Gallegas, Inv. Pesquera, 30: 561-576. Barcelona.

SEOANE-CAMBA, J. 1975. Características de la vegetación marina en la costa de Guipuzcoa, Anal. Inst. Bot. A.J. Cavanilles, 32 (1). Madrid.

VAN den HOEK, and DONZE, M. 1967. Algal Phytogeography of the european Atlantic coasts, Blumea, 15: 63-89. 